\title{
Valosin-Containing Protein (VCP/p97) Is a Potential Antiviral Target against Mononegavirales ${ }^{\dagger}$
}

\author{
Victor Latorre and Ron Geller * \\ Institute for Integrative Systems Biology (I2SysBio), Universitat de Valencia-CSIC, 46980 Valencia, Spain; \\ Victor.Latorre-Rosello@uv.es \\ * Correspondence: Ron.Geller@uv.es; Tel.: +34-963543187 \\ + Presented at Viruses 2020-Novel Concepts in Virology, Barcelona, Spain, 5-7 February 2020. \\ Published: 30 June 2020
}

\begin{abstract}
The viral order Mononegavirales consist of eight virus families. Members of these families include some of the most infectious (Measles, lethal (Ebola and Rabies), and most common viruses (Respiratory syncytial virus, RSV). Despite their medical importance, few vaccines and no antiviral treatments are available for treating infections with these viruses. Being obligate cellular parasites, viruses must rely on the cellular machinery for their replication. One example of this is the widespread use of molecular chaperones, which assist the correct folding of newly synthesized proteins, refold misfolded or aggregated proteins, and play key roles in maintaining proteostasis in cells. Targeting chaperones required for viral replication may, therefore, provide an antiviral approach. In this work, we set out to identify all the members of the cytoplasmic chaperone network that are involved in the replication of RSV using an RNA interference screen. Among our hits is valosin-containing protein (VCP; also known as p97), a chaperone involved in ubiquitin-mediated protein degradation, which has been shown to play a role in the life cycle of several viruses. We investigated the role of VCP during RSV and vesicular stomatitis virus (VSV) infections using specific VCP inhibitors. Our results suggest that VCP activity is necessary for RSV and VSV replication and may constitute a promising antiviral approach for the Mononegavirales.
\end{abstract}

Keywords: antivirals; Mononegavirales; valosin-containing protein; respiratory syncytial virus; vesicular stomatitis virus 